\title{
Socio-Economic Dynamics of Intra-Familial Elder Abuse in Baringo County, Kenya
}

\author{
Chesang' Consolata Jepkemei ${ }^{1}$, Samuel M. Mwangi ${ }^{2}$, Parvin Moloo ${ }^{3}$ \\ ${ }^{1}$ Student, Master of Arts in Sociology of Kenyatta University, Kenya \\ ${ }^{2}$ Senior Lecturer, Department of Sociology, School of Humanities and Social Sciences, Kenyatta \\ University, Kenya \\ ${ }^{3}$ Senior Lecturer, Department of Sociology, School of Humanities and Social Sciences, Kenyatta \\ University, Kenya
}

\section{ABSTRACT}

Until the emergence of policies and legal frameworks to address domestic violence -including intimate partner and child abuse- in the last decades of the $20^{\text {th }}$ century, abuse of the older people had persisted as a private matter that was accorded little public attention. Even so, intra-familial elder abuse (IFEA) has been acknowledged globally as a pervasive problem, associated with overwhelming distinct consequences, outcomes, and societal expenses. With an overall increase in the older persons' populace, IFEA is expected to become a more pressing issue, affecting masses of older individuals globally. Kenya is experiencing population aging at a high rate, which implies that, with it, elder abuse, and particularly IFEA is anticipated to become a more pressing problem, distressing millions of older individuals countrywide. IFEA refers to as a type of family violence has been defined as a sole or repeated mistreatment and/or abusive action, which can be an act of commission or omission, intentional and unintentional, towards older persons within the family context. The definition, conceptualization, and perceptions of intra-familial elder abuse vary across societies and culture, because, what may be deemed abusive in one society might not be the case in another, thus making the whole issue of elder abuse and particularly IFEA dynamic, with variations across boundaries, religions, economic, and social settings. This study sought to explore the socio-economic dynamics of IFEA in Baringo County. The study objectives were to profile the dominant types of IFEA, to examine the dynamics of IFEA in relation to the associated socio-economic risk factors, and to assess the dynamics associated with reporting and disclosure of IFEA, guided by Homan's Social Exchange theory. The study embraced a cross-sectional analytical survey design to collect both qualitative and quantitative data. The study was conducted in Baringo County. The respondents of the study were older persons in the area who were aged 70 years and older; 226 older persons from two purposively sampled sub counties were sampled for the study. The study also targeted key informants including local authorities, health care authorities, adult protection agency representatives and law enforcement. Semi-structured interviews, key informant interviews, and focus group discussion guides were used in data collection. Quantitative data was analyzed using the SPSS 21.0 to generate both descriptive and inferential statistics such as chi-square tests. Qualitative data was analyzed thematically. The study established that most prevalent form of intra-familial abuse reported in the study was psychological abuse (79.2\%) while sexual abuse was the least (19.9\%) prevalent type of IFEA. The study found out that gender of the victim, victim dependence and vulnerability, living arrangements, trust relationships, social isolation and financial dependency on the older person influenced the older persons' experiences with the different types of abuse reported in the study. Based on the reported dynamics of IFEA in relation to reporting, the study concluded that given the right platforms, older persons are more likely to report incidences of abuse. The study concludes that IFEA is a dynamic social problem, which varies across cultural contexts, 
perceptions, socio-economic risk factors, as well as in its reporting and disclosure. The study recommends education, sensitization, and public awareness campaigns at the community level as preventive strategies aimed at informing members of the society about IFEA, what it constitutes, and how it can be addressed.

Key Words: Elderly Abuse, Abuse Reporting, Socio Economic Dynamics of Intra-Familial

DOI 10.35942/ ijcab.v5i4.202

Cite this Article:

Chesang, C., Mwangi, S., \& Moloo, P. (2021). Socio-Economic Dynamics of Intra-Familial Elder Abuse in Baringo County, Kenya. International Journal of Current Aspects, 5(4), 1-19. https://doi.org/10.35942/ijcab.v5i4.202

\subsection{Introduction}

The abuse of older persons within the family setting dates back to ancient times (Pillemer, Burnes, Rifns \& Lachs, 2016). Until the emergence of policies and legal frameworks to address domestic violence (including intimate partner and child abuse) in the last decades of the twentieth century, abuse of the older individuals had remained a reserved matter that was accorded little public attention (Pillemer et al., 2016). However, intra-familial elder abuse (IFEA) in the recent decades, has been acknowledged globally as a universal and momentous problem, which necessitates the urgent consideration of health care systems, civil society organizations, policymakers, governments, and the civic at large (Von, Schiamberg \& Chee 2012). Overall, intra-familial elder abuse is associated with overwhelming individual consequences, outcomes, and societal costs, warranting consideration as a stern issue (Von et al. 2012). Research has shown that in contemporary society, household and family members commit the most violent crimes against older people (Jackson \& Hafemeister 2014)

Furthermore, with an overall increase in the older persons' populace, IFEA is projected to become a more persistent issue, affecting millions of older persons globally (Collins, 2018). According to WHO report of 2015, there will be a tremendous demographic transition, where, the global population of individuals aged 65 years and above is projected to grow in twofold from 900 million in 2005 to 2 billion by the year 2050 (United Nations Department of Economic and Social Affairs, $2013 ; 2014)$. The report also indicated that the vast majority of the older adults live in middle and low-income countries such as Latin America, Asia, and Africa. The doubling of this cohort of the population is attributed to longer lifespans resulting from improved medical care, improved diet, and nutrition, as well as improvement in the general wellbeing of the old people (Jackson \& Hafemeister 2014). However, the implication of population on elder abuse is that there will be an increased dependency of the older people on others, which then increases their vulnerability to abuse (Pillemer et al., 2016)

Recent and past statistics have evidenced the prevalence of elder abuse, as a form of family violence across the world. A series of studies conducted by the WHO (2016) indicated that China has the highest aggregated prevalence rate, which was attributed to the failure of the family members to accomplish their kinship obligations, dislocating older people as the heads of households, and depriving them of their independence, thus increasing their susceptibility to abuse. Overall, the research reported an accumulated intra-familial elder abuse pervasiveness ranging from $2.2 \%$ to $36.2 \%$, with an average of $14.3 \%$. Through all the studies, the uppermost aggregated pervasiveness was reported in China (36.0\%) and Nigeria (30.2\%), trailed by Israel (18.5\%), India 
(14.0\%), Germany (10.8\%), Spain (10.6\%), Mexico (10.6\%), United States (9.0\%), and Canada (4.5\%) (World Health Organization, 2016).

The common aspect among the studies carried out in these countries is that they recognized the underlying role and impact of cultural practices, norms, attitudes, and traditions, such as sexism, ageism, and the existence of a culture of violence, in propagating intra-familial elder abuse (Jackson \& Hafemeister 2014). Moreover, inequity and discrimination against the older person within the family due to individual attributes (such as gender, age, physical infirmities, sexuality and cultural background), were identified as the underlying social and health conditions for IFEA, alongside overt and subtle exploitation of power imbalances (National Council of Abuse, 2015). In a meta-analysis carried out in 28 countries in Africa, the prevalence rate was estimated at $11.6 \%$ for psychological abuse, $6.8 \%$ for financial abuse, $4.2 \%$ for neglect, $2.6 \%$ for physical abuse, and $0.9 \%$ for sexual abuse (Beard, Officer \& Cassels 2016). In most parts of Africa, including Kenya, the family is traditionally valued as the cradle of the care of the family needs, comprising those of the elderly. However, globalization, urbanization and modernization, westernization, and their accompanied cultures have weakened the traditional family, kinship, and community ties to provide support for the older persons (Mba, 2007). This is because of the changing family structures and power relations, which have then disadvantaged the elderly, exposing them to higher risks of abuse and mistreatment (Von et al., 2012).

British Scholars first described elder abuse, as a societal, political, and public health concern in 1975 as "granny battering" (Bustorn, 1975). The universal definition of elder abuse was put forward by the Action on Elder Abuse in 1993 as a "single, or repeated act, or lack of appropriate action, taking place within any relationship where there is an expectation of trust, which causes harm or distress to an older person or violates their human and civil rights" (Roberto, 2016). This definition was then adopted by the WHO and the International Network for Prevention of Elder Abuse (INPEA) in 2002 and is widely used across the world (Pillemer et al., 2016). However, the definition, conceptualization, and perceptions of elder abuse vary across societies and culture, because, what may be deemed abusive in one society, might not be the case in another, thus making the whole issue of elder abuse and particularly IFEA, dynamic, with variations across boundaries, religions, economic, and social settings (Jackson \& Hafemeister, 2014). To this point, therefore, it is evident that aging as a process in and of itself, varies across gender, family set-ups, regions, and cultures, and these variations dictate how society view and treat older persons, as well as determine what is considered abusive (United Nations Fund for Population Activities and HelpAge International, 2012). In this view, the socio-economic dynamics associated with IFEA have a significant role in influencing definitions, attitudes, and help-seeking behaviors, and in explaining the associated risk factors of intra-familial elder abuse. Even so, the intersection of socio-economic dynamics and IFEA has been captured scantly, thus merited further studies, particularly in Baringo County, which had no particular studies on it. This study, therefore, sought to examine the socioeconomic dynamics of intra-familial elder abuse in Baringo County, Kenya.

\subsection{Statement of the Problem}

Elder abuse within the family context is widely acknowledged as a pervasive and momentous issue that continues to affect millions of older persons globally. With the overall increase in the global older population's aging, future trends signify that family caregiving for senior older persons will become a progressively demanding responsibility for household and family members. The majorities of the older individuals live in multigenerational extended families and get informal care from their partners, adult children, and other members of the family. Older persons across the 
world are grappling with the various forms of IFEA at the hands of their immediate family and the community or society, and yet the issue remains an isolated matter, concealed from the public, leaving the older victims in susceptible conditions that lead to both physical and mental ill health (Collins, 2006). As a form of family violence, IFEA intensely affects a victim's physical and mental health (NCA, 2015). Generally, intra-familial elder abuse is associated with overwhelming physical consequences such as disability, injury, and worsened health statuses; psychological outcomes such as depression, loss of trust, dignity, hope, and anxiety; as well as social effects including social isolation, lack of social support and communication or even death.

Given that the significant majority of older Kenyans reside in domestic settings characterized by extended and multigenerational households, there is no doubt that most of the elder abuse incidences are perpetrated by family members who often are primary caregivers for the older adults. However, the definition, conceptualization, and perceptions of intra-familial elder abuse vary across societies and culture, because, what may be deemed abusive in one society might not be the case in another, thus making the whole issue of elder abuse and particularly IFEA dynamic, with variations across boundaries, religions, economic, and social settings. The intersection of socio-economic dynamics and IFEA, therefore, deserves further examination particularly in Baringo County, which to the best of my knowledge has no particular studies on it, with the aim of bringing an understanding of the scope and nature of IFEA in the study area from a Sociologic perspective. To get a greater understanding of these dynamics, therefore, this study sought to examine the socio-economic dynamics driving intra-familial elder abuse in Baringo County Kenya.

\subsection{Objectives of the Study}

The overall goal of this study was to examine the socio-economic dynamics of intra-familial elder abuse in Baringo County. The following were the specific objectives of the study:

1. To profile the socio-demographic characteristics of the older persons in Baringo County.

2. To profile the dominant types of intrafamilial elder abuse in Baringo County .

3. To examine the dynamics of intrafamilial elder abuse in relation to the associated socioeconomic risk factors in Baringo County.

4. To examine the dynamics associated with reporting and disclosure of intrafamilial elder abuse in Baringo County.

5. To suggest plausible recommendations to address the prevalence of intra-familial elder abuse.

\subsection{Literature Review}

\subsection{Theoretical Framework}

A theory is the construction of explicit accounts in explaining empirical findings on a social phenomenon (Bengston, Rice, and Johnson, 1999). In other words, a theory provides us with an explanation of what we observe empirically, which then leads to the creation of a knowledge base that guides further research, and understanding of a phenomenon. Therefore, a theoretical perspective provides us with a framework in which knowledge can explain and understood (Glaser \& Strauss 2017). A theory also produces testable hypotheses that can guide research. In summary, a theory can be termed as "a set of lenses through which we can view and make sense of what we observe in the inquiry." With this regard, this study was guided by the social exchange theory of 
aging in understanding the socio-economic dynamics of intra-familial elder abuse in Baringo County.

The Social Exchange Theory of is a sociological theory developed by George C. Homans in 1950. The theory supposes that persons in the social status quo choose actions that maximize their probability of fulfilling self-interests in those circumstances (Johnson, 1977). This supposition is based on three basic assumptions. Firstly, individuals are considered rational actors, who calculate the costs and benefits of social exchanges. Another assumption of the social exchange theory is that the people who engage in social interactions are logically seeking to maximize the rewards to be gained from those circumstances including material goods such as money, inheritance, and living arrangements, and non-material gains such as approval, status, and prestige (Gelles, 1999). Thirdly, exchange processes produce rewards for individuals, which lead to the modelling of social interactions that not only aid a person's needs but also restrain them in the way they may eventually seek to meet those needs (Gelles, 1999).

With regard to intra-familial elder abuse, as individuals grow old, the proportion of rewards to costs linked with their social interactions might change based on their social statuses (e.g. being an older person) and individual possessions (e.g. power, money, wealth, and the ability to work and offer care to others) (Abolfathi Momtaz, Hamid \& Ibrahim, 2013). In this case as the resources of the OPs decline with age (including income, health, and loss of employment or community roles); OPs are more possible to be in uneven and imbalanced social exchanges. The key element of the theory in this view is dependency. In reference to the SE theory, therefore, IFEA may ensue because of the victim's dependency on the perpetrator and vice versa. In this case, it is presumed that IFEA is the outcome of the older person's increasing reliance on the caregiver, who in most cases is a family member. Therefore, as people grow old, they become more vulnerable, feeble, and reliant on household and family members for support, which may increase their susceptibility to intra-familial elder abuse.

\subsection{Conceptual Framework}

A conceptual framework refers to a methodical tool, used to make and organize conceptual distinctions and ideas in a subsequent presentation, to assist a researcher to interlink concepts of a study. The conceptual framework for this study therefore, was constructed based on the literature review and presented in the figure below. In this framework, the independent variables are socioeconomic risk factors, and the dynamics of reporting and disclosure of IFEA. Each of these independent variables has particular sets of variable as presented in the conceptual framework above. The socio-economic risk factors are the variables that are responsible for driving the different types of intra-familial elder abuse. The dependent variable is intrafamilial elder abuse, for which the socio-economic dynamics is examined. Policy and legislative frameworks as well as education and advocacy interventions are the intervening variables in the study. 


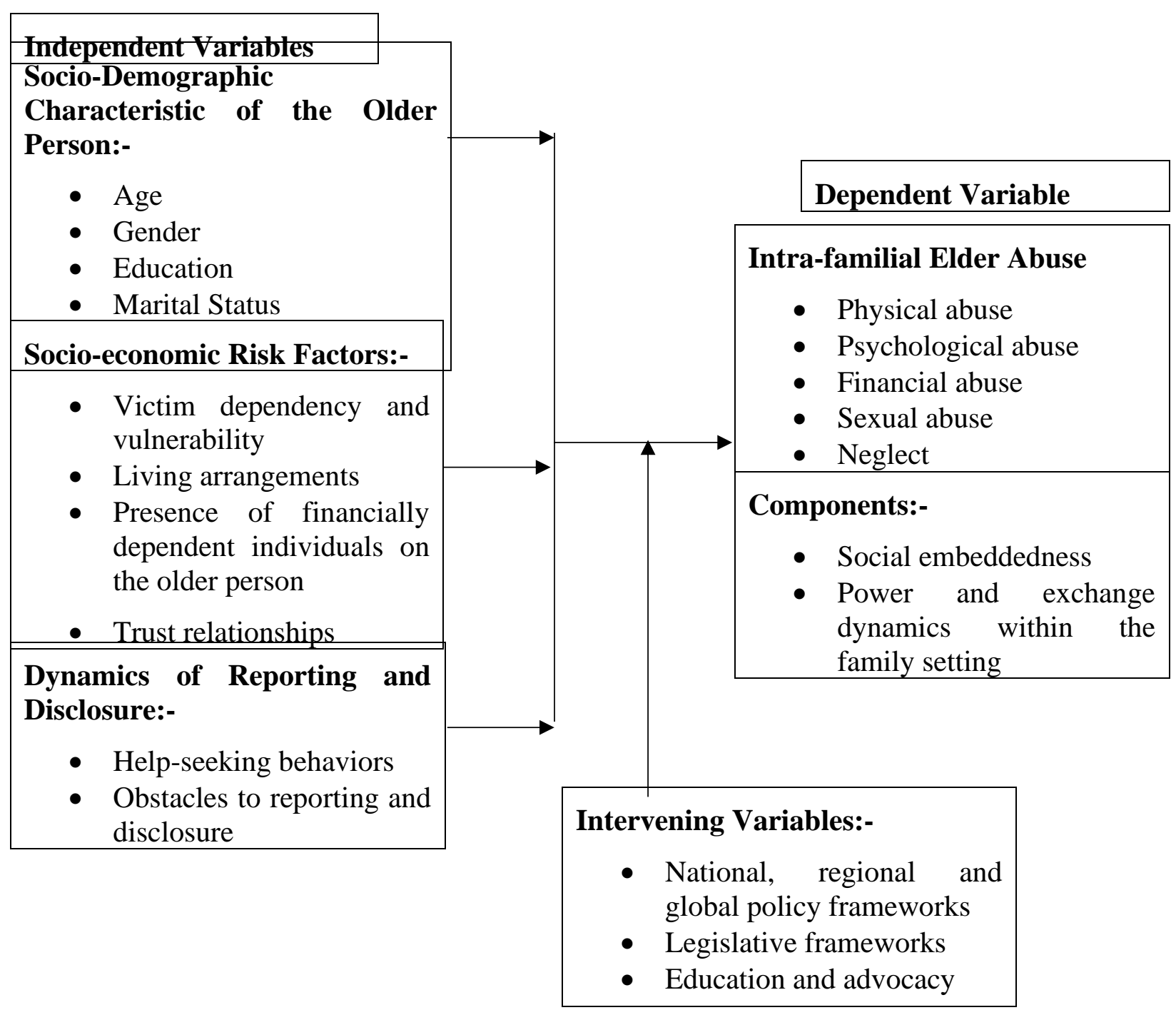

Figure 1: Conceptual Framework for the Study Source-Researcher

\subsection{Research Methodology}

This study adopted a mixed method approach, in which empirical qualitative and quantitative viewpoints, inference techniques, and methods were used to study intra-familial elder abuse in Baringo County. In particular, the study employed the cross-sectional analytical survey design to collect both qualitative and quantitative data. The rationale for this design is that it was significant in examining intra-familial elder abuse, and to analyze the questions of who, what, when, where, and how socio-economic dynamics are associated with intra-familial elder abuse in Baringo County (Small, Mario 2011). In essence, it was used to establish the causal relationship between socio-economic factors and IFEA in Baringo County. With regard to the time dimension, there was a one-time interaction with the participants of the study

The study was conducted in Baringo County, one of the 47 counties in Kenya, located in the Northern region of the prior Rift Valley Province. It borders West Pokot and Turkana Counties to the North, Nakuru County, and Kericho County to the South Samburu County and Laikipia County to the East, Elgeyo Marakwet County to the West and Uasin Gishu County to the South West . It 
covers an area of 8,655 square $\mathrm{km}$. The county has six constituencies: Baringo Central, Baringo South, Baringo North, Mogotio, Eldama Ravine, and Tiaty Constituency. The total population of Baringo County is 555,561 (GoK, 2010). However, the actual study was carried out in Baringo North and Baringo Central Constituencies. It is also important to note that, Baringo North constituency is primarily rural, while Baringo Central both rural and urban, and because the study focuses on IFEA both in the rural and urban family setups, it is expected that sufficient data was gathered and which was generalized to the entire population. The total area of Baringo North Constituency is 1,703.50 square kilometers, with a population of 93,789. On the other hand, Baringo Central has a total area of 588.52 square kilometers and a total population of 81, 480.

The target population of this study was older women and men, who are aged 70 years and older from Baringo North and Baringo Central Constituencies. The population of those aged 70 years and older in Baringo County is 18, 334 (Kenya Population Census, 2009). The number of OPs in North Baringo constituency is estimated at 3357, and 3150 in Baringo Central Constituency. The aggregate population of this study was approximately 6507 . The unit of analysis for this study was the older persons aged 70 years and older within households in the various wards of Baringo North and Central Constituencies. In addition, the families were another unit of analysis, whereby it was used to determine the family setup that the older persons lived in.

This study adopted a mixed sampling technique that comprised of both probability and nonprobability sampling techniques. Baringo North and Baringo Central Sub Counties were purposively sampled as the studies areas. The rationale for the purposive selection of these study areas was that Baringo North constituency is primarily rural, while Baringo Central both rural and urban, and because the study focuses on IFEA both in the rural and urban family setups, it was expected that sufficient data will be gathered and which could be generalized to the entire population. Baringo Central Sub County on the other hand has five (5) wards namely Kabarnet, Sacho, Tenges, Ewalel, and Kapropita Wards. Baringo North Sub County has five (5) wards namely Kabartonjo, Barwessa, Saimo-Kipsaraman, Saimo-Soi, and Bartabwa Wards. Four wards were randomly sampled for the study from both study sites - two from Baringo North and two from Baringo Central. Kabartonjo and Saimo-Soi wards were selected in Baringo North whereas Kabarnet and Kapropita wards were selected in Baringo Central study area. From each of the selected wards, two sub-locations were randomly sampled. Mosop and Kapkirwok Sub-Locations were selected in Kabartonjo Ward; Rondinin and Sibilo Sub locations in Saimo-Soi Ward; Seguton and Lelmen Sub locations were selected in Kabarnet Ward; and Riwo and Kaprogonya sub locations were selected in Kapropita Ward.

Proportionate sampling was used to distribute the study sample, where the sample was equally distributed across the two sub-counties, the four wards, and the eight sub-locations. A list of the older persons in the selected sub-locations was obtained from the assistant chiefs, from where the researcher randomly sampled the respondents for the study. The participants were randomly selected from lists of older persons who receive the Old Persons Cash Transfer (OPCT) Funds provided by the Kenyan government in the different locales. The local authorities from where the sampled participants resided provided this sampling frame. The usage of these sampling techniques guaranteed representativeness of the data that was obtained. Key informants and participants of the focus group discussions were selected using the purposive sampling technique.

The sample for this study was determined using the Black and Champion sample size determining formula, which assumes a descriptive statistic whose values from random sampling techniques would be normally distributed (Black \& Champion, 1976). The formula is as follows: 


$$
N=\left[\frac{\mathrm{Z} \delta}{\chi}\right]^{2}
$$

Where:

$\mathbf{N}$ is the desired sample size

$\mathrm{Z}$ is the standard score appropriate at $95 \%$ confidence interval

$\chi$ is the desired descriptive statistic

$\delta$ Is the estimate of the population standard deviation (estimated at 3.841 from literature of previously reviewed studies)

$$
\begin{aligned}
\therefore \mathrm{N} & =\left[\frac{(\mathbf{1 . 9 6})(\mathbf{3 . 8 4 1})}{\mathbf{0 . 5}}\right]^{2} \\
& =226 \text { older persons }
\end{aligned}
$$

Besides the older persons as respondents of the study, fourteen (14) key informants were also involved in the study. They comprised of two assistant county commissioners from each of the two sub counties, eight assistant chiefs each from the eight selected sub locations, two social workers, and two health care personnel from geriatric departments of the sub-county hospitals. With respect to the focus group discussions, four (4) focus group discussions, two in each sub county were conducted. The FGDs consisted eight members each, who were older persons aged 70 years and older and they were gender-inclusive. The members of the FGD were randomly selected.

The study used both quantitative and qualitative research methods namely: semi-structured interviews, focus group discussions, and key informant interviews. These tools are discussed in detail below. Since this study adopted a mixed method approach, quantitative and qualitative data were analyzed separately. The quantitative data was analyzed using the Statistical Package for Social Sciences (SPSS) Version 21 to generate both inferential and descriptive statistics that helped answer the topic and the objectives of the study. Chi-square tests were run using SPSS to assess the relationship between the socio-economic risk factors and the prevalence of each type of intrafamilial elder abuse at a $p$-value $<0.05$. Qualitative data from the answers of open-ended questions in the data from the K.I.Is and the FGDs were coded and the repeated themes identified. The thematic analysis was used in line with the study objectives. The quantitative data was presented in the form of tables, while the qualitative in verbatim quotes.

\subsection{Data Analysis Results}

\subsection{Dominant types of intra-familial elder abuse}

There are five dominant types of elder abuse identified in the existing literature and they include physical, financial, psychological, sexual abuse, and the passive form of neglect (Yon et al., 2017). The prevalence rates of these typologies of IFEA as found out in the study are as follows: psychological abuse (at 79.2\%), financial abuse (73.5\%), neglect $(62.8 \%)$, physical abuse $(50.9 \%)$, and sexual abuse (19.9\%). In order to get an in-depth comprehension and insights on how each of these typologies of IFEA were manifested in the study area, various forms and indicators of each type of abuse were examined. 
Based on the different forms of psychological abuse identified in the study, the study found that most of the respondents experienced witchcraft allegations, threats with deprivation and institutionalization, as well as intimidation by individuals in their families. These forms of psychological abuse establish in the study are consistent with what is documented in existing literature regarding psychological elder abuse. Kabole, Kioli, and Onkware (2013) referred to the most common forms of psychological abuse reported which included witchcraft allegations, intimidation, isolation, and continued threatening of the older persons.

The study established that financial abuse included the OPs being coerced a family member(s) and/or caregiver to sign a property transfer document, title deed or financial documents against their will, land grabbing, and forceful taking or misusing of the older persons' money and other material possessions. Existing literature has also pointed out similar forms of elder abuse within the domestic settings. Collins (2018) indicated that the common forms of financial abuse of the older persons within the family include coercing the older person to sign financial and property transfer documents forcefully, forcing them to give property or stealing their possessions. In addition, the study also established that the most prevalent forms of neglect abuse as a type of IFEA in the study area include refusal by family members to take of the OPs, denying the older persons their prescribed medication, and OP being left unattended for too long. In addition, the study also established other forms of neglect including not being given medical attention when they are ill and although not very prevalent, the denial of basic needs including proper clothing, food, and water. Previous empirical studies undertaken referred to failure to provide basic needs for the OP, refusal of family members to look after the OP, especially those with physical infirmities and denying them necessary medical attention as the most prevalent forms of neglect of the older persons in domestic settings (Yunus, Hairi \& Choo, 2019).

For physical abuse, the study found that the most prevalent forms included actions that inflicted physical pain on the victim such as slapping, striking or hitting as well as physical restraining through being locked up in a room or being tied down. This finding conforms with the literature regarding the common forms of physical abuse which include physical restraining of the older person and actions that inflict physical pain such as slapping and so on (Clarysse et al., 2018). Lastly, the study examined sexual abuse, and it was found that the prevalent forms of this type of IFEA included sexually abusive acts such as touching the OP in a sexually abusive manner, being shown pornographic materials against their will and non-consensual sex. This finding agrees with the literature by Flores and colleagues (2019) which points out that the most prevalent forms of sexual abuse as a type of elder abuse include rape, indecent assault, sexual harassment or any behaviour and/or act towards the OP that is sexually abusive.

\subsection{Socio-economic risk factors of intra-familial elder abuse}

In the existing literature, it is widely acknowledged that a number of socio-economic risk factors drive the prevalence of elder abuse. The commonly cited risk socio-economic risk factors for intrafamilial elder abuse included those that range from victim risk factors to perpetrator risk factors. Various studies on elder abuse have also pointed out on the role played by victim-perpetrator relationships in increasing the susceptibility of the older persons to abuse. These factors include gender of the victim, victim dependence and vulnerability, living arrangements, trust relationships, and financial dependency on the older person among others (Bows \& Penhale, 2018; Fang \& Yan, 2018; Gil et al., 2015; Jeon et al., 2019; Santos et al., 2020, 2016). The study found that gender was one of the risk factors associated with the prevalence of IFEA in the study area. Based on the findings in the study, it was found out that the experiences of IFEA varied across gender with some 
types of IFEA being more prevalent among the older male population and others among the older female population. It was found that older men in the study were more susceptible to physical abuse and neglect as compared to the older women. This finding agrees with what is in literature that older men, rather than older women tend to be targets of neglect and physical abuse in the household settings (Ananias, et al. 2016).

On the other hand, the study established that older women rather than senior men in the study area tend to be targets of psychological, financial, and sexual abuse within the household setups. This finding is consistent with existing literature. Gil and colleagues (2015), Giraldo-Rodriguez and Rosas-Carrasco (2016), Lowenstein et al., (2009), and Naughton and colleagues (2010) pointed out that women are more probable than men to experience psychological, sexual, and financial abuse as prevalent types of intra-familial elder abuse. Additionally, the finding aligns with literature by Yon et al. (2017) which revealed that in most cultures around the world where women generally have inferior or lower social status, older women are at higher risks of sexual abuse and financial exploitation, especially seizing of their property when they are widowed.

The study examined victim dependency and vulnerability as risk factors for IFEA in terms of the older persons' need of aid in carrying out their activities of daily living (ADLs). The study established that victim dependency and vulnerability majorly due to physical infirmity and functional dependency did not increase the older persons' susceptibility to the various typologies of abuse in their domestic settings. Majority of the respondents who experienced the different types of abuse needed no assistance with ADLs. These findings reveal that whether the respondents needed assistance with ADLs or not, it did not have a direct implication on their possibility of encountering elder abuse within their family settings. This finding agrees with those of Pillimer and colleagues (2016), which indicated that the functional dependence of the OP did not increase their risks of being vulnerable to elder abuse. It however contrasts with the finding of a study by Fang and Yan (2018), Robson and colleagues (2010), and WHO (2020) which indicated that, across different societies, physical infirmity and functional dependency among the older persons has constantly been found to be associated with greater risk of abuse.

In the study, living arrangements as a likely risk factor was examined in terms of the older persons' ownership of residence and availability of private spaces/rooms in their residences. On ownership status of the OPs residence, the study examined prevalence of abuse among older persons who lived in three categories of residences i.e. own, rented, and someone else's. Based on these categories, the study established that ownership of residence was not directly associated with the older persons' experiences with intra-familial elder abuse since, regardless of the ownership status of the older persons' places of residence, physical abuse, psychological abuse, sexual abuse; financial abuse and neglect were significantly prevalent among the older persons in the study. In essence, the study found that ownership of residence has no effect on the older persons' experiences with IFEA since there is no statistically significant difference in experiences with abuse between those who reported abuse and those who reported no abuse across the three ways of living arrangements as identified in the study area.

In terms of the availability of private rooms for the older persons in their residences, the study established that that most of the older persons who had a private or personal room, did not experience physical abuse as compared to those who had no personal room, whom majority had experienced this type of abuse. In the contrary, the study also established that cases of neglect were higher among older persons, who did not have a personal room, as compared to those who had a personal room. These findings align with the literature that living arrangements, mostly lack of 
privacy for the older person has been associated with conflict in families and more so intra-familial elder abuse (Bows \& Penhale, 2018).

On the other hand, the study found that availability or lack of a private/personal room for the older person was not directly associated with the prevalence of psychological abuse, sexual abuse and financial abuse in Baringo County. This is based on the finding that these typologies of IFEA were prevalent among older persons with a personal room. This implies that availability or lack of a private/personal room for the older person is not directly associated with the prevalence of psychological abuse. This finding contradicts literature by Mose and Gillum (2016), NCA (2016) and WHO (2019) which indicates that living arrangements, more specifically lack of privacy for the OP, is associated with conflict within families and more so prevalence of elder abuse within the family settings.

Existing literature has evidenced that trust relationships between the older persons and the people they live with as a risk factor associated with the prevalence of elder abuse within the family contexts (Ervin and Henderson, 2020; Bows and Penhale, 2018). In examining the association between trust and IFEA the study established that most of the older persons who reported cases of physical abuse, psychological, financial, sexual abuse, and neglect, indicated that they trusted the people they lived. This finding is consistent with those of a study by Ervin \& Henderson (2020) and Pillimer and colleagues (2016) which revealed that elder abuse manifests when a family member or caregiver who is trusted by the older person to have control over his/her medical and financial decisions and to care and protect them, takes advantage of the position by abusing the OP. In conclusion, that trusting people they lived with increased the older persons' risks of being abused by the family member and/or caregiver.

In the study, the presence of economically dependent individuals on the older persons was established to be a risk factor for psychological, financial, sexual abuse, and neglect and not a likely risk factor for physical abuse. This is based on the findings that most of the older persons, who had financially dependent individuals within their family settings, had experienced these typologies of IFEA as compared to those who did not. This finding is congruent with of a study by SANTOS ET AL., 2020 (2016), which revealed that in most instances; abusers are economically reliant of the victims (the OPs) for accommodation, maintenance and other financial expenditures. According to the research findings, the existence of a web of interdependency between the perpetrator and the victim increase the risks of elder abuse. In most of the cases of their study, it was found out that the abuser was financially dependent on the abused. Findings of studies by Straus and colleagues (2017), Von, and colleagues (2012) which reported that elder abuse perpetrators were considerably more dependent on the victim for financial assistance and housing also confirm this finding. The study therefore concludes that the presence of a financially dependent person the older person is a risk factor associated with the OPs experiences with intrafamilial elder abuse.

\subsection{Dynamics of Intra-familial Elder Abuse in Relation to Reporting and Disclosure}

The study examined the dynamics of IFEA in relation to reporting and disclosure. These dynamics included: the possibility of the OPs to report abuse incidences identified in the previous section; the relationship between the possibility of reporting and gender; the possibility of reporting the different types of IFEA; the possible reasons for reporting the abuse incidences; the obstacles to abuse reporting; and to whom the older persons were likely to report the abuse cases. To begin with, the study established that majority of the older persons in the study were likely to report the 
IFEA situations they had experienced within the family settings. In examining the relationship between the possibility or reporting the IFEA incidences and gender with the aim of understanding gender differences in reporting and disclosure, the study found that most of the female older persons were likely to report abuse while most male older persons were likely not to report the abuse incidences.

This can be attributed to the socialization of male and females in the community in a way male are generally expected to be strong and not express feelings of "weakness" while females are generally viewed as "emotionally weak." These gender stereotypes and cultural expectations may therefore hinder help-seeking behaviours for IFEA among the older males. This finding is consistent to those of studies by Dow and colleagues (2019) which revealed that stereotypes including the man being the protector and provider for the family, and socio-cultural norms that necessitate men to demonstrate strength and resilience could dissuade older men from conceding, seeking help, and reporting cases of abuse that they have encountered in the family setting. It is also congruent to literature by Cohen and colleagues (2007) which indicates that even when older men report elder abuse incidences, there are not always sufficient social care services to support them. Moreover, in examining the possibility of the respondents to report the different types of intra-familial elder abuse reported in the study, it was found that the majority of the respondents who encountered financial abuse, were likely to report and disclose their encounters. Majority of those who experienced physical, psychological, sexual abuse and neglect indicated that they were not probably to report the abuse cases. These findings agree with those of literature by Jackson and Hafemeister (2018) and HealthLink BC (2020) which indicates that financial abuse is the most commonly reported type of elder abuse. This is because majority of the OPs are vulnerable to financial abuse, they rely on family and caregivers to assist them with financial matters, and most of them lack knowledge on their finances.

Regarding the possible reasons for reporting, the participants in the study pointed out a number of reasons. They include the feeling of being confident that they will be supported and helped, the sense of relief after reporting, the hope of living in safer environments and the belief that the benefits of telling someone about the abuse cases would outweigh its potential costs. Others pointed out that they would report because they thought an injustice had occurred to them, and others would report because they know whom to turn to for help. Concerning the potential obstacles and reasons for not reporting IFEA incidences, the study established a number of reasons. Most of the respondents indicated that they would not report due to the fear that it might ruin their relationships with their families, the fear of judgement, and the fear that situations might get worse after reporting. Additionally, the study also established that victims of IFEA were not likely to report and disclose their experiences since they were not aware of where or whom to turn to for help as well as the fact that they would fear the potential consequences of disclosing. These finding is consistent with Jackson \& Hafemeister's (2015) arguments that the dynamics of the victim and abuser relationships play a significant role in EA reporting and disclosure. Both studies reveal that the web of mutual dependency and emotional attachment between the victim and the perpetrator has in many occasions hindered the older persons from reporting their experiences of abuse by family members and caregivers. Lastly, regarding whom to report the abuse incidences to, the study found that most of the respondents indicated that they would report the abuse to a family member, with others indicating to report to a trusted friend, a religious leader, the police, a medical provider and the least indicating to report to the adult protection services. These findings reveal that as much as the main perpetrators of IFEA are family members and caregivers within the family setting, most of the OPs still prefer to resolve the matter with a family member than to report the 
cases to the police or even the adult protective agencies. These findings are consistent with findings from a study by Jackson and Hafemeister (2015) that the victims of elder abuse are hesitant to seek for help because they see the abuse incidences as family matters especially when it involves a family member or caregiver.

\subsection{Conclusions and Recommendations}

\subsection{Conclusions}

The study on the socio-economic dynamics of intra-familial elder abuse captured data on the older person's experiences of psychological, physical, sexual, financial abuse and neglect within intrafamilial relationships by family members and paid caregivers. In profiling the prevalence of these typologies of abuse, it was established that psychological abuse was the most prevalent $(79.2 \%)$ followed by financial abuse $(73.5 \%)$, neglect $(62.8 \%)$, physical abuse $(50.9 \%)$, and the least prevalent was sexual abuse (19.9\%). The study found that the prevalence rates of abuse varied across different risk factors including gender, victim vulnerability, living arrangements, financial dependence of family members on the older persons and trust relationships. The specification of risk factors of IFEA in objective two is significant in providing a rational basis for elder abuse prevention programs and strategies.

The significant risk factors for physical abuse included the Ops need for assistance with ADLs and the trust relationships between the OP and family members and/or caregivers. For psychological abuse, the primary socio-economic risk factors included the Ops need for assistance with ADLs, the trust relationships between the OP and family members and/or caregivers and the presence of financially dependent individuals within the family setting on the OP. In respect to the risk factors associated with financial abuse, the study found trust relationships and presence of financially dependent individuals as the key factors. Additionally, the key risk factors for sexual abuse included ownership status of the gender of the victim, older person's residence and trust relationships. Lastly, for neglect, the study established that the key risk factors related to it included availability of a private or personal room for the OP, trust relationships, and presence of financially dependent individuals within the family setting on the OP.

Additionally, in examining the dynamics of intra-familial elder abuse in relation to reporting and disclosure, the study concludes that given the right platforms, older persons are more likely to report incidences of abuse. It is important to note that in examining reporting and disclosure, it was a question of "possibility" of reporting and this does not reflect the true representation of IFEA cases reported in the study area. However, for this to be achieved, various effective mechanisms ought to be put in place to address the possible barriers to reporting and disclosure, thus protecting the older persons from the severe effects of elder abuse. Majority of the older individuals the study lived in multi-generational extended households and got informal care from their partners, adult children, and other members of the family. Moreover, it was evident that elder abuse was difficult to be talked about by the victims especially where the perpetrators are family members. Overall, based on the findings of this study, the study concludes that IFEA is a severe public health, economic, and social issue affecting many older persons across all socio-economic groups in the society. This study findings contributed to an understanding of the experiences of the older persons with elder abuse within the family settings and its findings and recommendations can be of significance to practice and policy through designing and suggesting policy interventions to address this form of domestic and family violence. In understanding this social problem, the 
members of the society will be more vigilant in reporting cases of elder abuse thus helping eradicate and prevent future incidences.

\subsection{Recommendations}

The study recommends a critical evaluation of existing interventions aimed at addressing elder abuse as a form of family violence in order to ensure their efficacy in addressing the issue. Examples of existing interventions include legal interventions such as state adult protection; community-based interventions such as medical and psychosocial needs programs; and education and abuse prevention interventions like empowerment and advocacy campaigns. The study also recommends that governments, both national and county initiate evaluations of their existing reporting laws that address elder abuse to determine how early case uncovering of IFEA leads to improved outcomes for the victims. Due to poor record keeping practices observed in the study, it is recommended that adult social service providers need to develop safeguards to reinforce their documentation of intra-familial elder abuse both in group and individual records, notwithstanding to whether the abuse incidences are reported to the authorities. The study also recommends social work extension services to include the protection of older adults who are victims of IFEA, as it has been proven in existing literature as one of the most promising interventions for elder abuse in domestic settings.

\section{References}

Aboderin, I. (2012). Ageing Africa: opportunities for development. Global Population Ageing: Peril or Promise?, 161(242), 69.

Abolfathi Momtaz, Y., Hamid, T. A., \& Ibrahim, R. (2013). Theories and measures of elder abuse. Psychogeriatrics, 13(3), 182-188.

Acierno, R., Lawyer, S. R., Rheingold, A., Kilpatrick, D. G., Resnick, H. S., \& Saunders, B. E. (2007). Current psychopathology in previously assaulted older adults. Journal of Interpersonal Violence, 22(2), 250-258.

Aday, R. H., Wallace, J. B., \& Scott, S. J. (2017). Generational differences in knowledge, recognition, and perceptions of elder abuse reporting. Educational Gerontology, 43(11), 568-581.

Adib, M., Esmaeili, M., Zakerimoghadam, M., \& Nayeri, N. D. (2019). Barriers to help-seeking for elder abuse: A qualitative study of older adults. Geriatric Nursing.

Ananias, J., \& Strydom, H. (2014). Factors contributing to elder abuse and neglect in the informal caregiving setting. Social Work, 50(2), 268-284.

Ananias, J., Salonika, L. A., Black, N., \& Strydom, H. (2016). Social work engagement in the community-based care of older people in Namibia. The Handbook of Social Work and Social Development in Africa, 145.

Anetzberger, G. J. (2005). The reality of elder abuse. Clinical Gerontologist, 28(1-2), 1-25.

Australian Network for the Prevention of Elder Abuse (2018). Psychological Abuse. Retrieved from: https://www.eapu.com.au/uploads/ANPEA/ANPEA

Baker, P. R., Francis, D. P., Hairi, N. N., Othman, S., \& Choo, W. Y. (2016). Interventions for preventing abuse in the elderly. Cochrane Database of Systematic Reviews, (8).

Beard, J. R., Officer, A. M., \& Cassels, A. K. (2016). The world report on ageing and health.

Beard, J. R., Officer, A., de Carvalho, I. A., Sadana, R., Pot, A. M., Michel, J. P., \& Thiyagarajan, J. A. (2016). The World report on ageing and health: a policy framework for healthy ageing. The Lancet, 387(10033), 2145-2154. 
Bengtson, V. L., Rice, C. J., \& Johnson, M. L. (1999). Are theories of aging important? Models and explanations in gerontology at the turn of the century. Handbook of theories of aging, 3-20.

Berger, R. (2017). Aging in America: Ageism and general attitudes toward growing old and the elderly. Open Journal of Social Sciences, 5(08), 183.

Black, J. A., \& Champion, D. J. (1976). Methods and issues in social research. John Wiley \& Sons.

Bobitt, J., Carter, J., \& Kuhne, J. (2018). Advancing national policy on elder abuse. Public Policy \& Aging Report.

Bows, H., \& Penhale, B. (2018). Elder abuse and social work: Research, theory and practice.

Bryman, A. (2016). Social research methods. Oxford university press.

Burnes, D., Acierno, R., \& Hernandez-Tejada, M. (2018). Help-seeking among victims of elder abuse: findings from the National Elder Mistreatment Study. The Journals of Gerontology: Series $B$.

Burston, G. R. (1975). Granny-battering. British Medical Journal, 3(5983), 592.

Cannell, B., Gonzalez, J. M. R., Livingston, M., Jetelina, K. K., Burnett, J., \& Weitlauf, J. C. (2019). Pilot testing the detection of elder abuse through emergency care technicians (DETECT) screening tool: results from the DETECT pilot project. Journal of elder abuse \& neglect, 1-17.

Chokkanathan, S., \& Lee, A. E. (2005). Elder mistreatment in urban India: A community based study. Journal of Elder Abuse \& Neglect, 17, 45-61. doi:10.1300/J084v17n02_03

Clarysse, K., Kivlahan, C., Beyer, I., \& Gutermuth, J. (2018). Signs of physical abuse and neglect in the mature patient. Clinics in dermatology, 36(2), 264-270.

Cohen, M., Levin, S. H., Gagin, R., \& Friedman, G. (2007). Elder abuse: disparities between older people's disclosure of abuse, evident signs of abuse, and high risk of abuse. Journal of the American Geriatrics Society, 55(8), 1224-1230.

Collins, J. (2018). Financial abuse of older persons: A criminal law perspective. In Ageing, Gender and Family Law (pp. 61-75). Routledge..

Conrad, K. J., \& Conrad, K. M. (2018). Abuser risk measure: Reports by alleged victims to adult protective services. The Gerontologist.

Cotterell, E., Leonardi, S., Coward, D., Thomson, J., \& Walters, A. (2015). Elder abuse in the ACT: A literature review. Canberra: COTA (Council on the Ageing) ACT.

de Lima, M. P., Vergueiro, M. E., Gonzalez, A. J., Martins, P., \& Oliveira, J. G, (2018). Relations between Elder Abuse, Ageism and Perceptions of Age. Retreived from: https://www.researchgate.net/publication/324394124

Di Rosa, Melchiorre, Quattrini, \& Lamura. (2015). Mistreatment of older persons in Europe and in Italy Results from the ABUEL study. CAREGIVER DAY 2015 Prevenire 1'Abuso verso gli Anziani Carpi (MO). Carpi. Available online at: http://www.combatingelderabuse.eu/ wp-content/uploads/2014/02/Di_Rosa_EN.pdf

Doron, I., Brown, B., \& Somers, S. B. (2013). International protection for the human rights of older people: History and future prospects. In Ageism and mistreatment of older workers(pp. 165-179). Springer, Dordrecht.

Dow, B., Gahan, L., Gaffy, E., Joosten, M., Vrantsidis, F., \& Jarred, M. (2019). Barriers to Disclosing Elder Abuse and Taking Action in Australia. Journal of Family Violence, 1-9.

Ervin, S., \& Henderson, E. (2020). Elder Abuse Victimization: What We Know from Researchand Practice-Based Evidence. 
Ezeh, A., Chepngeno, G., Kasiira, A., \& Woubalem, Z. (2006). The situation of older people in poor urban settings: the case of Nairobi, Kenya. Aging in sub-Saharan Africa: Recommendations for furthering research, 189-213.

Fang, B., \& Yan, E. (2018). Abuse of older persons with dementia: a review of the literature. Trauma, Violence, \& Abuse, 19(2), 127-147.

Ferreira, M. (2005). Elder abuse in Africa: What policy and legal provisions are there to address the violence?. Journal of Elder Abuse \& Neglect, 16(2), 17-32.

Flores, R. J., Campo-Arias, A., Stimpson, J. P., Chalela, C. M., \& Reyes-Ortiz, C. A. (2018). The association between past sexual abuse and depression in older adults from Colombia. Journal of geriatric psychiatry and neurology, 31(1), 13-18.

Fowler, J. (2018). Preparing Psychology Doctorate Students to Identify and ReportSuspected Elder Abuse: Evaluating the Effectivenessof Elder Abuse Training (Doctoral dissertation, The Wright Institute).

Garre-Olmo, J., Planas-Pujol, X., López-Pousa, S., Juvinyà, D., Vilà, A., Vilalta-Franch, J., \& Frailty and Dependence in Girona Study Group. (2009). Prevalence and risk factors of suspected elder abuse subtypes in people aged 75 and older. Journal of the American Geriatrics Society, 57(5), 815-822.

Gelles, R. (1999). Through a sociological lens: Social structure and family violence. Sociology of Families, 299-308.

Gil, A. P., Kislaya, I., Santos, A. J., Nunes, B., Nicolau, R., \& Fernandes, A. A. (2015). Elder abuse in Portugal: Findings from the first national prevalence study. Journal of Elder Abuse \& Neglect, 27, 174-195. doi:10.1080/08946566.2014.953659

Giraldo-Rodríguez, L., \& Rosas-Carrasco, O. (2013). Development and psychometric properties of the Geriatric Mistreatment Scale. Geriatrics \& Gerontology International, 13, 466-474. doi:10.1111/j.1447-0594.2012.00894.x

Glaser, B. G., \& Strauss, A. L. (2017). Discovery of grounded theory: Strategies for qualitative research. Routledge.

Gorman, M. (2017). Development and the rights of older people. In The ageing and development report (pp. 21-39). Routledge.

Government of Kenya. (2010). 2009 Kenya Population and Housing Census. Nairobi: Government Printers.

Hamby, S., Smith, A., Mitchell, K., \& Turner, H. (2016). Poly-victimization and resilience portfolios: Trends in violence research that can enhance the understanding and prevention of elder abuse. Journal of Elder Abuse \& Neglect, 28(4-5), 217-234.

Hassan, A. M., \& Sakwa, M. (2018). Effects of Cash Transfer Programme on Older Persons' wellbeing in Garissa County, Kenya'. International Journal of Social Sciences and Information Technology, 4(3).

Hays, W. L. (1973). Statistics for social scientists. SF: Holt, Rinehart \& Winston.

Heale, R., \& Forbes, D. (2013). Understanding triangulation in research. Evidence-Based Nursing, ebnurs-2013.

HealthLink BC (2020). Abuse and Neglect of Older Adults: Understanding Gender Differences. Elder Abuse Prevention Series 93c. Retrieved from: https://www.healthlinkbc.ca/healthlinkbc-files/older-adult-abuse-gender

Help Age International, 2013. Sustainable Development in an Ageing World: A call to UN Member States on the development agenda beyond 2015. 
International Network for the Prevention of Elder Abuse (INPEA) (2016). Abuse of the Elderly. Retrieved from: http://www.inpea.net/images/Elder_Abuse_Fact_Sheet.pdf

Jackson, S. L., \& Hafemeister, T. L. (2015). The impact of relationship dynamics on the detection and reporting of elder abuse occurring in domestic settings. Journal of elder abuse \& neglect, 27(2), 121-145.

Jackson, S. L., \& Hafemeister, T. L. (2018). Detecting and Reporting Four Types of Elder Abuse: How Official Adult Protective Services Reports Obscure Older Adults' Self-Efficacy. $J$ Aging Gerontology Res, 1(1), 101.

Jeon, G. S., Cho, S. I., Choi, K., \& Jang, K. S. (2019). Gender differences in the prevalence and correlates of elder abuse in a community-dwelling older population in Korea. International journal of environmental research and public health, 16(1), 100.

Jeon, G. S., Cho, S. I., Choi, K., \& Jang, K. S. (2019). Gender differences in the prevalence and correlates of elder abuse in a community-dwelling older population in Korea. International journal of environmental research and public health, 16(1), 100.

Johnson, W. T. (1977). Exchange in perspective: the promises of George C. Homans. Behavioral theory in sociology, 49-90.

Kabole, A. L., Kioli, F. N., \& Onkware, K. (2013). The social context of abuse of elderly people in Emuhaya District, Kenya. Sociology and Anthropology, 1(2), 76-86.

Kim, E. H. W., \& Cook, P. J. (2009). Child-to-parent financial transfers and their contribution to reducing elder poverty in Korea. Family Support Networks and Population Ageing, 61.

Levy, S. R., \& Macdonald, J. L. (2016). Progress on understanding ageism. Journal of Social Issues, 72(1), 5-25.

Liu, P. J., Conrad, K. J., Beach, S. R., Iris, M., \& Schiamberg, L. B. (2017). The importance of investigating abuser characteristics in elder emotional/psychological abuse: results from adult protective services data. The Journals of Gerontology: Series B.

Lowenstein, A., Eisikovits, Z., Band-Winterstein, T., \& Enosh, G. (2009). Is elder abuse and neglect a social phenomenon? Data from the first national prevalence survey in Israel. Journal of Elder Abuse \& Neglect, 21, 253-277. doi:10.1080/08946560902997629

Matcha, D. A. (2007). The sociology of aging: An international perspective. Sloan Publishing.

Mba, C. J. (2007). Elder abuse in parts of Africa and the way forward. Gerontechnology, 6(4), 230-235.

Melchiorre, M. G., Chiatti, C., Lamura, G., Torres-Gonzales, F., Stankunas, M., Lindert, J., ... \& Soares, J. F. (2013). Social support, socio-economic status, health and abuse among older people in seven European countries. PloS one, 8(1), e54856.

Mose, G. B., \& Gillum, T. L. (2016). Intimate partner violence in African immigrant communities in the United States: Reflections from the IDVAAC African women's round table on domestic violence. Journal of Aggression, Maltreatment \& Trauma, 25(1), 50-62.

Mukherjee, D. (2011). Organizational structures of elder abuse reporting systems. Administration in Social Work, 35(5), 517-531.

Mysyuk, Y., Westendorp, R. G., \& Lindenberg, J. (2013). Added value of elder abuse definitions: a review. Ageing research reviews, 12(1), 50-57.

Näre, L., Walsh, K., \& Baldassar, L. (2017). Ageing in transnational contexts: transforming everyday practices and identities in later life. Identities, 24(5), 515-523.

National Council for Population development, (2016). A Policy Brief on the Increasing Number of Old People. Retrieved from: http://www.ncpd.go.ke/wpcontent/uploads/2016/11/Policy-Brief-54 
National Council of Aging (2015). Prevalence and Underreporting of Elder Abuse in the United States. Retrieved from: https://www.ifa-fiv.org/partner-profiles/national-council-for-theelderly-ncoa/

National Social Protection Secretariat (2016). Retrieved from: http://www.socialprotection.or.ke/

Norris, D., Fancey, P., Power, E., \& Ross, P. (2013). The critical-ecological framework: advancing knowledge, practice, and policy on older adult abuse. Journal of elder abuse \& neglect, 25(1), 40-55.

Ondigi, A. N., \& Ondigi, S. R. (2012). The influence of poverty and well being of the elderly people in Nyanza Province, Kenya. Asian Social Science, 8(2), 211.

Ostan, R., Monti, D., Gueresi, P., Bussolotto, M., Franceschi, C., \& Baggio, G. (2016). Gender, aging and longevity in humans: an update of an intriguing/neglected scenario paving the way to a gender-specific medicine. Clinical science, 130(19), 1711-1725.

Patterson, M., \& Malley-Morrison, K. (2006). A cognitive-ecological approach to elder abuse in five cultures: human rights and education. Educational Gerontology, 32(1), 73-82.

Perkins, N. H., Spira, M., \& Key, J. E. (2018). Intergenerational Transmission of Physical and Emotional Sibling Violence: A Potential Connection to Elder Abuse. Families in Society, 99(3), 256-268.

Pillemer Karl, David Burnes, Catherine Rifn and Mark S. Lachs (2016). Elder Abuse: Global Situation, Risk Factors, and Prevention Strategies, Gerontologist, Vol. 56, No. 2, pp. 194205 co.

Republic of Kenya (2010). The Kenyan Constitution: Care and Protection of Older Members of Society Bill. Government Press.

Republic of Kenya (2014). Sessional Paper No. 2 of 2014 on the National Policy on Older Persons and Aging. Government Press.

Roberto, K. A. (2016). The complexities of elder abuse. American Psychologist, 71(4), 302.

Robson, M. G., Dong, X., \& Simon, M. A. (2010). Is impairment in physical function associated with increased risk of elder mistreatment? Findings from a community-dwelling Chinese population. Public Health Reports, 125(5), 743-753.

Roger, K. S., Brownridge, D. A., \& Ursel, J. (2015). Theorizing low levels of reporting of abuse of older immigrant women. Violence against women, 21(5), 632-651.

Santos, A. J., Nunes, B., Kislaya, I., Gil, A. P., \& Ribeiro, O. (2019). Elder abuse victimization patterns: latent class analysis using perpetrators and abusive behaviours. $B M C$ geriatrics, 19(1), 117.

Santos, M. A. B. D., Moreira, R. D. S., Faccio, P. F., Gomes, G. C., \& Silva, V. D. L. (2020). Factors associated with elder abuse: a systematic review of the literature. Ciência \& Saúde Coletiva, 25, 2153-2175.

Schoeni, R. F., Martin, L. G., Andreski, P. M., \& Freedman, V. A. (2005). Persistent and growing socioeconomic disparities in disability among the elderly: 1982-2002. American journal of public health, 95(11), 2065-2070..

Small, Mario L. 2011. "How to Conduct a Mixed Method Study: Recent Trends in a Rapidly Growing Literature.” Annual Review of Sociology 37:55-84

Storey, J. E. (2020). Risk factors for elder abuse and neglect: A review of the literature. Aggression and violent Biolent behaviorBehavior, 50, 101339.

Straus, M. A., Gelles, R. J., \& Steinmetz, S. K. (2017). Behind closed doors: Violence in the American family. Routledge.

Thane, P. (2003). Social histories of old age and aging. Journal of Social History, 37(1), 93-111. 
UNDESA, 2013. World Population Prospects: the 2012 revision.

UNDESA. 2014. Population ageing and sustainable development. UNDESA Population Division. 2012. Population Ageing and Development 2012, Wall Chart.

UNFPA and Help Age International, 2012. Ageing in the Twenty-First Century: A Celebration and a Challenge. London, 2012.

United Nations, Department of Economic and Social Affairs, Population Division, 2015. World Population Ageing. New York.

United Nations, U. (2011). Current Status of the Social Situation, Well-Being, Participation in Development and Rights of Older Persons Worldwide. New York.

Von Heydrich L. Schiamberg L. B., \& Chee G. (2012). Social-relational risk factors for predicting elder physical abuse: An ecological bi-focal model. International Journal of Aging \& Human Development, 75, 71-94. doi:10.2190/AG.75.1.f [PubMed]

World Health Organization (2019). What is elder abuse? Action on Elder Abuse Bulletin, 125-145. Retrieved

from: https://www.who.int/violence_injury_prevention/violence/global_campaign/en/chap5.pdf

World Health Organization (June 2020). Elder abuse. Global Strategy and Action Plan on Ageing and Health. Retrieved from: https://www.who.int/news-room/fact-sheets/detail/elderabuse

World Health Organization, (2002). Proposed working definition of an older person in Africa for the MDS Project. Retrived from: https://www.who.int/healthinfo/survey/ageingdefnolder/en/

World Health Organization. (2017). WHO clinical consortium on healthy ageing: topic focus: frailty and intrinsic capacity: report of consortium meeting, 1-2 December 2016 in Geneva, Switzerland (No. WHO/FWC/ALC/17.2). World Health Organization.

Yaffe, M. J., \& Tazkarji, B. (2012). Understanding elder abuse in family practice. Canadian family physician, 58(12), 1336-1340.

Yon, Y., Mikton, C. R., Gassoumis, Z. D., \& Wilber, K. H. (2017). Elder abuse prevalence in community settings: a systematic review and meta-analysis. The Lancet Global Health, 5(2)e147-e156.

Yunus, R. M., Hairi, N. N., \& Choo, W. Y. (2019). Consequences of elder abuse and neglect: a systematic review of observational studies. Trauma, Violence, \& Abuse, 20(2), 197-213.

This is an open-access article published and distributed under the terms and conditions of the (c) (7) \&) Creative Commons Attribution 4.0 International License of United States unless otherwise stated. Access, citation and distribution of this article is allowed with full recognition of the authors and the source. Authors seeking to publish with an Internationally Peer Reviewed Journals should consider https://www.ijcab.org/ by writing to the Editor at editor@ijicab.org or submitting online at https://journals.ijcab.org/journals/index.php. The articles must be quality and meet originality test.

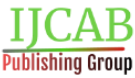

